\title{
A Microfounded Herding Model and Its Estimation On German Survey Expectations
}

\author{
Reiner Franke
}

\begin{abstract}
The paper considers the dynamic adjustments of an average opinion index that can be derived from a microfounded framework where the individual agents switch between two kinds of sentiment with certain transition probabilities. The index can thus represent a general business climate, i.e., expectations about the future course of the economy. This approach is empirically tested with the survey expectations published by the $Z E W$ and ifo institute. The estimated coefficients make economic sense and are highly significant. In particular, besides effects from fundamental data like the output gap in the recent past, one can identify a strong herding mechanism within both panels, such that the agents do not just join the majority but, metaphorically speaking, follow each single motion of the crowd. In addition, the transition probabilities of the $Z E W$ agents are found to be influenced by the ifo climate but not the other way around.
\end{abstract}

JEL classications: $D 84, E_{32}, E_{37}$

Keywords: herding dynamics, animal spirits, individual transition probabilities, ifo and ZEW climate index, Kalman filter

* University of Kiel, Germany. I wish to thank Thomas Lux and Xiaokang Wang for helpful discussions and the reestimation of the key results with their alternative, more ambitious econometric approach. I also thank two anonymous referees for their comments which, in particular, helped to streamline the presentation. For a more detailed version of the paper see the list of references. Financial support from EU STREP ComplexMarkets, contract number 516446, is gratefully acknowledged.

\section{Correspondence Address:}

Dr. Reiner Franke, Department of Economics, University of Kiel,

e-mail: franke@ikfs.uni-bremen.de

Received I7 June 2007, accepted 2I April 2008

(C) INTERVENTION 5 (2), 2008, 30I-328 


\section{Introduction}

While homogeneous rational expectations are still the ruling paradigm in macroeconomic theory, expectations in the real world are far more diversified and may approximate rational expectations, if at all, only in the aggregate. Being sufficiently self-critical, insecure, and uncertain of the future events, the individual agents are eager to learn about the expectations of others, or about a general climate that is currently prevailing. This is the reason why real-world agents, and financial markets in particular, closely monitor the periodic publications of economic survey indicators. ${ }^{\text {. }}$

The evaluation of survey expectations is usually concerned with their ability to predict the future course of the economy. Thus, in the case of Germany and the four surveys available for this country, the ZEW and the ifo expectations indices are held to show the best performance regarding economic growth (see, e.g., Broyer/Savry 2002), where the ZEW index could be praised to have the highest correlation with industrial production when it is leading five to six months, vis-à-vis the ifo expectations with a lead of three or four months (Stadler 200I, Hüfner/Schröder 2002a und 2002b). On the other hand, what is lacking in these discussions is a conceptual framework that describes how an opinion index may be formed and how it adjusts over time; with a particular view to possible herding effects of the responding subjects where, for example, optimism feeds optimism. The topic is of more than remote theoretical interest since a pronounced herding mechanism would run counter the abovementioned rational expectations.

One step in the direction of a better understanding of the factors driving the survey expectations is a study by Lahl and Hüfner (2003) on the ZEW indicator of economic sentiment. Using ordinary least squares they find that besides a few lags of this variable itself, also the German manufacturing order data, the German term structure, and the US consumer condence indicator have some additional explanatory power. ${ }^{2}$ The dynamic adjustments of the ZEW indicator can thus be described as a combination of self-reference, as represented by the significant autoregressive coefficients in the estimation, and of hetero-reference (Orléan 1989). The latter expression means the state of opinion of a social group in its relationship to an external norm, which is here given by a set of central macroeconomic variables in the real, financial and foreign sector.

The investigation by Lahl and Hüfner helps identify basic components in the determination of a survey index. Nevertheless, despite the motivation behind the selection of the explanatory variables, the regression equation is not yet a structural theory. Although it might be tempting to interpret the autoregressive coefficients as reflections of a herding effect, the structure in the regression equation is too poor to warrant such a conclusion.

I It may here also be noted that in contrast to macroeconomic theory which almost exclusively focusses on inflationary expectations, these surveys mainly relate to economic activity as a whole.

2 Taking up the previous footnote, we nd it remarkable that among the other independent variables that the more practically oriented authors explored, they neither included an inflation variable nor a real rate of interest. 
Alternatively, the coefficients may just as well result from some inertia in the adjustment process, or the index itself may move quite in line with other economic variables that are relevant to the survey participants.

This is where the present paper sets in. It takes up a more than 20 year old approach by Weidlich and Haag (1983), which derives the evolution of an aggregate sentiment variable from the probabilistic interactions of individual heterogeneous agents at the micro level. While they build up their model within the framework of statistical mechanics, it is here reformulated in a less technical way such that we can finally arrive at an ordinary adjustment equation at the macro level. Since the basic elements of the original approach are maintained, this sentiment dynamics can be said to have a rigorous microfoundation. ${ }^{3}$

More specifically, our model easily captures the self-referential and hetero-referential mechanisms mentioned above. It also admits of a clear specification of the idea of herding, which recently has found increasing attention in verbal descriptions of sentiment dynamics.

The sentiment adjustment equation is a very convenient tool that can be readily and fruitfully incorporated into many (non-orthodox) macrodynamic models with a role to beliefs, business expectations, or the famous sanimal spiritsı. In the present paper, however, we focus on an empirical validation of this approach, where the ZEW and ifo expectation indices seem most suitable to test the model's claim that it can explain the changes in an aggregate sentiment index. That is, we estimate the model to see if its parameters can be idenfied and come out with the economically meaningful signs. On the other hand, significant results in this respect may help us understand the dynamics of the two indices and reveal the features they have in common or in which they differ. As has already been alluded to, the recognition of a possible herding component will then be of particular interest.

The remainder of the paper is organized as follows. Section 2 begins with a short overview of the historical background of the model put forward here. It then introduces the transition probabilities of the individual agents which govern their switches between two alternative attitudes, derives an adjustment equation for the aggregated attitudes, i.e. for the general climate index, and considers several feedback variables with which the transition probabilities may vary. Section 3 presents the data and estimates the basic model by nonlinear least-squares. Here special care has to be taken to solve a problem of overdetermination. Section 4 contains two parts. The first one examines whether the transition probabil-

3 We are aware that in contemporary macroeconomics the expression $>$ micro usually stands for intertemporal optimization, while in other respects micro and macro collapse into the representative agent which is then called 'microfoundation . By contrast, the present approach is related to the original meaning of micro in that it explicitly considers many individual agents with diffierent attitudes. Insofar as we propose a stylized description of how these attitudes are changing and derive an adjustment equation for the aggregate outcome, we feel fully entitled to characterize this type of macrodynamics as being 'microfounded, too. In our view, usage of this term should not narrow down to denote just one very specific modelling strategy, even if it is the dominating one with a certain claim to absoluteness. 
ities of the ZEW agents are also influenced by the ifo climate index and vice versa. In the second part an additional variable is introduced which is unobservable and to some extent can capture the effects that have so far been omitted. This generalization of the model is estimated by the (extended) Kalman filter. Section 5 concludes.

\section{The Dynamic Adjustment Equation of the Climate Index 2.I Historical background}

As indicated in the introduction, the model of sentiment dynamics that we propose originates with a stimulating book in the social sciences by Weidlich and Haag (1983). Unfortunately, their approach has not found its way into contemporary macroeconomic theory, although for (heterodox) economists working with feedback-guided macrodynamic systems it would have been an exceptionally fruitful design. In our opinion, two reasons are responsible for this neglect. First, the formulation of the model does not only refer to a probabilistic framework, its analysis also uses concepts from the theory of statistical mechanics like the master equation and the Fokker-Planck equation which are largely unknown to many economists. They are used to study definite time paths of aggregate variables, whereas statistical mechanics is concerned with the evolution of an entire probability distribution or at least, in the mean field approximations, with the time path of expected values. Even the latter concept, however, can be hard to assess, namely, if the stochastic equilibrium of the system is characterized by a bimodal probability density function. In this (otherwise most appealing) case, expected values would become meaningless in predicting the likely value of a variable.

A second aspect is insufficient marketing. While the approach was (also) employed in a number of macroeconomic papers, the topics they dealt with were somewhat detached or "exotic" (Kraft et al. 1986, Haag et al. 1987, Weise/Kraft 1988), or the ordinary reader probably soon drowned in a sea of specification details, so that he or she could no longer get hold of the attractive essence of the approach (Weidlich/Braun 1992). Nevertheless, macroeconomists with a wider area of interest could have also learned from several related articles by, in particular, Kirman (1993), Lux (1995, 1997 and 1998), or Orléan (1995), all of which appeared in highly reputable journals which most of them will have browsed on a regular basis. In sum, the approach by Weidlich and Haag (1983) or similar formulations in the I990s offered macroeconomists a good chance to introduce sentiment dynamics into their models in a very convenient, even standardized way, but this chance was largely missed. ${ }^{4}$ By contrast, it is interesting to add that similar ideas, although specified in different ways, have become quite popular over the last ten years in agent-based models of financial markets (see Hommes 2006, for an overview).

4 Taylor and O'Connell (1985), Franke and Asada (1994), and Flaschel et al. (1997, chapter I2) are three of the few macrodynamic contributions whose central expectational variable is an economy- 
Taking up the original specification by Weidlich and Haag, we contend that for our present purpose the whole statistical mechanics apparatus could be dispensed with. Instead, in that language, we can concentrate on a self-contained derivation of the Langevin equation. Accordingly, an ordinary stochastic or deterministic, difference or differential equation will emerge which can subsequently be analyzed, simulated, or estimated like any other adjustment equation of this type.

\subsection{From Microscopic Transition Probabilities to a Macroscopic Adjustment Equation}

Consider a fixed population of $2 N$ agents where at time $t$ each agent is either optimistic or pessimistic about the future prospects of the economy. Designating an optimistic and pessimistic attitude by $(+)$ and $(-)$, respectively, let $n_{t}^{+}, n_{t}^{-}$be the number of optimistic and pessimistic agents at $t\left(n_{t}^{+}+n_{t}^{-}=2 N\right)$. Next, put $n_{t}=\left(n_{t}^{+}+n_{t}^{-}\right) / 2$ and define $x_{t}=n_{t} / N$. All agents having equal weight in the population, this ratio is the average attitude of agents or, as we will call it, the climate index. Clearly, $-1 \leq x_{t} \leq 1$; optimism and pessimism balance in a state $x_{t}=0$; and at $x_{t}>0\left(x_{t}<0\right)$ optimistic (pessimistic) agents form a majority.

Agents may change their attitude over time. We model this in discrete time and slice time into adjustment periods of length $\Delta t>0$. That is, the agents' attitudes are considered at time $t, t+\Delta t, t+2 \Delta t$, etc. The individual changes will depend on a great variety of idiosyncratic circumstances, which one will not want to specify in all of their details. It rather seems suitable to introduce random elements in this respect, in order to keep the modelling simple and to avoid arbitrary assumptions. Therefore, the basic concept to describe the changes in the climate index are the transition probabilities of the individual agents: at time $t$, let $\pi_{t}^{-+}$be the probability per unit of time that an agent changes from pessimistic to optimistic, and $\pi_{t}^{+-}$the probability for an opposite change. More exactly, $\Delta t \pi_{t}^{-+}$is the probability that an agent who is pessimistic at $t$ has become optimistic at the next point in time $t+\Delta t$; and likewise $\Delta t \pi_{t}^{+-}$for an optimistic agent. ${ }^{5}$ These probabilities are uniform across the population. They are, however, not fixed but are influenced by the variations of certain macro variables, which will be discussed further below.

Let us beforehand examine how, given $\pi_{t}^{+-}$and $\pi_{t}^{-+}$, the climate index changes from $t$ to $t+\Delta t .^{6}$ To this end we consider the 'excess' index of optimistic agents $n_{t}=\left(n_{t}^{+}-n_{t}^{-}\right) / 2$.

wide business climate, which is there called a state of confidence. The dynamic adjustments of the latter, however, were formalized in an ad-hoc manner. Without essentially affecting the final results, this part of the models could be easily, and conceptually more satisfactorily, reformulated along the lines propounded in the present paper. Hence, the implicit criticism of not having been sufficiently alert to a fruitful and innovative idea in the past also falls back on the author of this paper, especially since he knew of the article by Weise and Kraft (1988) and Lux (1995) already quite early.

5 Which does not rule out that an agent switches several times within this adjustment period, although this might not appear very plausible for periods of moderate length $\Delta t$.

6 The following argument draws on Alfarano and Lux (2007: appendix Ai and A2). 
It rises by 1 if a pessimistic agent becomes optimistic (when $n_{t}^{+}$increases and $n_{t}^{-}$decreases by 1 ), and it declines by 1 if an optimistic agent turns pessimistic. Denoting by $c_{t}^{-+}$and $c_{t}^{+-}$the number of converts from pessimism to optimism and vice versa, respectively, we have

$$
n_{t+\Delta t}=n_{t}+c_{t}^{-+}-c_{t}^{+-}
$$

As the number of pessimistic agents at time $t$ can be written as $n_{t}^{-}=N-n_{t}$, the number $c_{t}^{-+}$of agents turning optimistic can be viewed as arising from $N-n_{t}$ random draws each of which has a probability $\Delta t \pi_{t}^{-+}$for the event ${ }^{+}+1<$ (and the complement for the no-change event $(0)$ ). The number of these events are then added up. Hence, the random variable $c_{t}^{-+}$has a binomial distribution $B\left(N-n_{t}, \Delta t \pi_{t}^{-+}\right)$. Analogously, $n_{t}^{+}=N+n_{t}$ being the number of optimistic agents in $t$, the random variable $c_{t}^{+-}$is distributed as $B\left(N+n_{t}, \Delta t \pi_{t}^{+-}\right) .^{7}$

The expected values of these variables are $E\left(c_{t}^{-+}\right)=\left(N-n_{t}\right) \Delta t \pi_{t}^{-+}$and $E\left(c_{t}^{+-}\right)=$ $\left(N+n_{t}\right) \Delta t \pi_{t}^{+-}$, their variances amount to $\operatorname{Var}\left(c_{t}^{-+}\right)=\left(N-n_{t}\right) \Delta t \pi_{t}^{-+}\left(1-\Delta t \pi_{t}^{-+}\right)$and $\operatorname{Var}\left(c_{t}^{+-}\right)=$ $\left(N+n_{t}\right) \Delta t \pi_{t}^{+-}\left(1-\Delta t \pi_{t}^{+-}\right)$. If the expected values are large enough (exceeding 5 or Io), the binomial distributions are (very) well approximated by the Gaussian distributions with the same first and second moments. Taking for granted that the population is large and $n_{t}$ not too close to the boundaries $\pm N$, eq. (I) becomes

$$
\begin{aligned}
n_{t+\Delta t}-n_{t} & =c_{t}^{-+}-c_{t}^{+-}=E\left(c_{t}^{-+}\right)-E\left(c_{t}^{+-}\right)+\sqrt{\operatorname{Var}\left(c_{t}^{-+}\right)} \xi_{t}^{+}-\sqrt{\operatorname{Var}\left(c_{t}^{+-}\right)} \xi_{t}^{-} \\
& =E\left(c_{t}^{-+}\right)-E\left(c_{t}^{+-}\right)+\sqrt{\operatorname{Var}\left(c_{t}^{-+}\right)+\operatorname{Var}\left(c_{t}^{+-}\right)} \xi_{t}, \quad \xi_{t} \sim N(0,1)
\end{aligned}
$$

where $\xi_{t}^{+}$and $\xi_{t}^{-}$are two independent random draws from the standard normal distribution $N(0,1)$ (with mean zero and variance equal to one). The last equality in (2) results from the fact that the difference between two normal distributions $\xi_{t}^{+}$and $\xi_{t}^{-}$is a normal distribution again: its mean is the difference between the two single means (i.e. zero) and its variance the sum of the two single variances. Referring to the climate index $x_{t}=$ $n_{t} / N$, we have $E\left(c_{t}^{-+}\right)-E\left(c_{t}^{+-}\right)=\Delta t \cdot\left[\left(1-x_{t}\right) \pi_{t}^{++}-\left(1+x_{t}\right) \pi_{t}^{+-}\right] \cdot N$ and $\operatorname{Var}\left(c_{t}^{-+}\right)+\operatorname{Var}\left(c_{t}^{+-}\right)=$ $\Delta t \cdot\left[\left(1-x_{t}\right) \pi_{t}^{-+}\left(1-\Delta t \pi_{t}^{-+}\right)+\left(1+x_{t}\right) \pi_{t}^{+-}\left(1-\Delta t \pi_{t}^{+-}\right)\right] \cdot N$. It thus remains to divide (2) by $N$, which yields

$$
\begin{aligned}
x_{t+\Delta t} & =x_{t}+\Delta t \cdot\left[\left(1-x_{t}\right) \pi_{t}^{-+}-\left(1+x_{t}\right) \pi_{t}^{+-}\right]+\left[\left(\sqrt{\Delta t D_{t}} / \sqrt{N}\right)\right] \xi_{t} \\
D_{t} & :=\left(1-x_{t}\right) \pi_{t}^{-+}\left(1-\Delta t \pi_{t}^{-+}\right)+\left(1+x_{t}\right) \pi_{t}^{+-}\left(1-\Delta t \pi_{t}^{+-}\right), \quad \xi_{t} \sim N(0,1)
\end{aligned}
$$

7 A binomial distribution $B(m, \pi)$ is the probability distribution for the number of successes $(k)$ in a sequence of $m$ independent sucess/failure experiments, each of which yields success with probability $\pi$. The probability of getting exactly $k$ successes is given by $\left({ }_{k}^{m}\right) \pi^{k}(1-\pi)^{m-k}$, the mean is $m \pi$, and the variance $m \pi(1-\pi)$. To be clear, we have presupposed that the individual agents are autonomous, i.e., the realizations of their opinion switching as they are induced by the transition probabilities occur independently of each other (which, depending on the specific social context and its network structure, might not be completely obvious). 
Equation (3) abstracts from the many individual and accidental switches in the agents' attitudes and summarizes them in a macroscopic stochastic equation that governs the changes in the climate index. It is the so-called Langevin equation that was announced above (here specified in discrete time). This relationship is usually derived by first setting up the entire probability distribution $P=P[x(t), t ; z(\cdot)]$ of $x$ at time $t$, possibly given the time path of a set of exogenous variables $z$. To analyze the rate of change of $P$ the powerful tool of the Fokker-Planck equation (FPE) is employed, which is itself a second-order approximation. Regarding (3), the intimate connection between FPE and the Langevin equation is shown by the fact that the first term in square brackets is the drift coefficient and $D_{t}$ corresponds to the fluctuation or diusion term in FPE. ${ }^{8}$

On the other hand, if one is not interested in the distribution $P$ and its evolution over time, the concept of FPE could be circumvented altogether and the story leading to eq. (3) may fully suffice. In fact, the assumptions required for (3) to be valid are not essentially stronger than those underlying the derivation of FPE.

Three special cases to which the adjustment equation (3) gives rise are easily recognized. First, the noise level decreases with the size of the population and in the limit $N \rightarrow \infty$, the sentiment dynamics becomes a deterministic process (provided $\pi_{t}^{-+}, \pi_{t}^{+-}$do not, directly or indirectly, increase with $N$ ). Second, the continuous-time limit $\Delta t \rightarrow 0$ is welldefined, too. If (3) is written as $x_{t+\Delta t}=x_{t}+A \Delta t+\tilde{D} \xi \sqrt{\Delta t}$, then this equation corresponds to the stochastic differential equation $d x=A d t+\tilde{D} W$, where $W$ is a normalized Brownian motion. Lastly, with $A=A(x, z)$ in this equation, an infinitesimally short adjustment period $\Delta t$, and an infinitely large population, the adjustments in (3) 'degenerate' to an ordinary differential equation $\dot{x}=A(x, z) .{ }^{9}$ Note that especially the deterministic cases, taken on their own or when incorporated into a more comprehensive framework, could be analyzed like any other difference or differential equation. These remarks show the wide scope of eq. (3) for macrodynamic modelling. All will then hinge on the specification of the transition probabilities, to which we now turn.

\subsection{Feedbacks in the Individual Transition Probabilities}

Generally, the transition probabilities $\pi_{t}^{+-}$and $\pi_{t}^{-+}$between $t$ and $t+\Delta t$ will change in response to the variations of a set of several variables that the agents observe. To ease the exposition, let us summarize the variables in a single feedback index $f_{t}$, which can attain positive and negative values in different stages the economy goes through. Positive and negative are related to the probability $\pi_{t}^{-+}$of switching from pessimistic to optimistic, that is, an increase in the feedback index $f_{t}$ increases $\pi_{t}^{-+}$and decreases the complementary probability $\pi_{t}^{+-}$.

8 See Weidlich and Haag (1983: 22-26) for a succinct presentation of the relationship between FPE and the Langevin equation in continuous time. An example of this treatment in discrete time is Alfarano et al. (2005: 23f., 46f.)

9 More scrupulously, first $N \rightarrow \infty$ and then $\Delta t \rightarrow 0$; or $N$ tends faster to infinity than $\Delta t$ to zero, such that $\Delta t / N \rightarrow 0$. 
It is an obvious concept, which Weidlich and Haag (I983) have also found very helpful in their formal analysis, to assume that the changes of the transition probabilities depend on the changes of the index $f_{t}$ in a linear way. More precisely, the relative changes, so that we have $d \pi_{t}^{-+} / \pi_{t}^{-+}=\alpha d f_{t}$ for some positive constant $\alpha$. By suitably scaling $f$, this constant can be set equal to one. Symmetry is another natural assumption to make, which gives us $d \pi_{t}^{+-} / \pi_{t}^{+-}=-d f_{t}$. Introducing $\nu>0$ as an integration constant', the specification of the transition probabilities reads ('exp being the exponential function),

$$
\pi_{t}^{-+}=\pi_{t}^{-+}\left(f_{t}\right)=\nu \exp \left(f_{t}\right), \quad \pi_{t}^{+-}=\pi_{t}^{+-}\left(f_{t}\right)=\nu \exp \left(-f_{t}\right)
$$

Certainly, (4) ensures positive values of the probabilities. The supplementary condition that the feedback index is bounded such that the probabilities are less than unity should be a property of the model into which (4) is incorporated, or the outcome of an empirical estimation.

A special feature of $(4)$ is $\pi_{t}^{-+}=\pi_{t}^{+-}=\nu>0$ when $f_{t}=0$. Hence even in the absence of active feedback forces, or when the different feedback variables neutralize each other, the agents will still change their attitude with a positive probability. These reversals, which can occur in either direction, are to be ascribed to idiosyncratic circumstances; they appear as purely random from a macroscopic point of view and should cancel out in the aggregate. For nonzero values of the feedback $f_{t}$, the coefficient $v$ measures the general responsiveness of the transition probabilities to the arrival of new information. So $v$ can be generally characterized as a flexibility parameter (Weidlich and Haag, I983: 4I).

While eq. (4) provides a first and useful organizational device, the meaningfulness of the model hinges essentially on the variables that are included in the feedback index. For a basic specification, we concentrate on two variables which, we think, are the most elementary ones to consider. The empirical validity of the model is constituted by estimations in the confines of this setting. Significant results are here also important if we want to sell our approach as a building block ready for implementation in a more encompassing macrodynamic framework, where the user will appreciate a parsimonious specification. Although adding further variables in the feedback index may be informative for specific purposes (two special issues will be examined later in Section 4), in general one will easily face the problem of arbitrariness: What will be the reason for enriching the feedback index just by this, but not another, variable?

The two variables whose influence on the transition probabilities we investigate are the climate index $x_{t}$ itself and a measure of economic activity as a whole. For the latter the concept of the output gap $y_{t}$ is employed, i.e. the percentage deviation of actual aggregate output from trend (or 'potential ) output. We do not only consider the levels of the two variables but, in order to capture possible momentum effects, also their rates of change. In this respect, let us now fix the time unit as well as the adjustment period as a month, $\Delta t=1$ [month]. Since the agents may guard against the noise that monthly variations can contain, changes over one or several months for $x_{t}$ and $y_{t}$ are allowed to enter the feedback index, where the corresponding lags $\tau_{x}$ and $\tau_{y}$ may be distinct. Thus, in a formulation (and 
dating) that is directly suited for estimation, our dynamic model of the business climate $x_{t}$ reads as follows:

$$
\begin{aligned}
x_{t} & =x_{t-1}+\nu\left[\left(1-x_{t-1}\right) \exp \left(f_{t-1}\right)-\left(1+x_{t-1}\right) \exp \left(-f_{t-1}\right)\right]+\varepsilon_{x, t} \\
f_{t-1} & =\phi_{0}+\phi_{x} x_{t-1}+\phi_{\Delta x} \Delta_{\tau_{x}} x_{t-1}+\phi_{y} y_{t-1}+\phi_{\Delta y} \Delta_{\tau_{y}} y_{t-1} \\
\Delta_{\tau_{u}} u_{t} & =\left(u_{t}-u_{t-\tau_{u}}\right) / \tau_{u} \quad \text { for } u=x, y
\end{aligned}
$$

Note that unlike the stochastic perturbations in eq. (3), which represent intrinsic noise at the micro level, the random terms $\varepsilon_{x, t}$ in (5) are assumed to have a constant standard deviation. Actually, the factor $1 / \sqrt{N}$ in (3) is typically so small that we will neglect the intrinsic noise. The present equation (5) rather conceives the $\varepsilon_{x, t}$ as primarily representing random forces from outside our theoretical framework, i.e. extrinsic noise.

For conceptual reasons another type of randomness should be mentioned here, which concerns the feedback index entering (5). These are possible "measurement errors «. In the first instance the term means that the agents, even if their information sets were identical, do not observe the same data as that used in an estimation of $(5)-(7)$. It suffices to touch on the two most important discrepancies: (I) the period- $(t-1)$ macro data may not have been available to the agents at that time or, if so, the data are likely to have been revised by the statistical authorities in the meantime; (2) as will become clear below, the agents had to determine the output gap in different ways from the econometrician.

It would therefore be appropriate to include a second random influence $\varepsilon_{f, t-1}$ in eq. (6) for the feedback index, which might also be serially correlated. Since, however, a corresponding amendment would require a more elaborated estimation procedure, we postpone this device until later in Section 4.2. For the time being, any such term $\varepsilon_{f, t-1}$ is omitted in (6) and we bear in mind that if these effects were relevant, they would be captured by the $\varepsilon_{x, t}$ perturbations, though possibly not in a fully adequate form.

Accepting the limitation to two explanatory variables, the composition of the feedback index is straightforward. Note first that the output gap $y$ as well as its rates of change $\Delta y$ are centered around zero. This allows us to interpret $\phi_{o}$ as a predisposition parameter, since in a neutral state where $x_{t-1}=\Delta_{\tau} x_{t-1}=y_{t-1}=\Delta_{\tau} y_{t-1}=0$, a positive $\phi_{o}$ gives rise to a probability $\pi_{t}^{-+}$of switching from pessimistic to optimistic that exceeds $\nu=\nu \cdot \exp (0)$, while the reverse probability $\pi_{t}^{+-}$is less than $\nu .{ }^{\text {Io }}$

Referring to the expressions that were already mentioned in the introduction, the feedback of the climate index on itself can be said to represent the aspect of self-reference or, in more topical language, the notion of herding, while the impact of the output gap on the

Io Weidlich and Haag (1983: 4I) call their counterpart of $\phi_{0}$ a preference parameter. Incidentally, a predisposition of the agents towards optimism does not necessarily imply that optimism dominates pessimism in a stationary state of the adjustment equation (5) (under $y_{t-1}=\Delta_{\tau} y_{t-1}=0$ ). In fact, this will depend on the coefficient $\phi_{x}$ : a stationary point $x^{*}=0$ of (5) for $\phi_{o}=0$ is shifted upward by a rising $\phi_{o}$ if $0<\phi_{x}<1$, and this $x *$ shifts downward if $\phi_{x}>1$; see Weidlich and Haag (1983: 42-44) and eq. (IO) further below. 
climate index is a hetero-referential mechanism. Regarding the herding component in the model, we would like to underline that including $x_{t-1}$ and $\Delta_{\tau} x_{t-1}$ in eqs (5), (6) admits an explicit structural interpretation with an immediate psychological plausibility, in contrast to the more technical autoregressive coefficients in the estimation approach to the climate

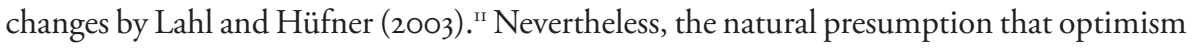
and pessimism are self-reinforcing, which would be reflected by positive coefficients $\phi_{x}$ or/ and $\phi_{\Delta x}$, will still have to be verified by the empirical estimations.

In finer detail, specification (6) distinguishes two variants of herding. A positive coefficient $\phi_{x}$ means that the probability of switching from pessimism to optimism is higher, and the reverse probability of switching from optimism to pessimism is lower, the more agents have already converted to an optimistic attitude. The herding effect expressed by $\phi_{x}>0$ may thus be characterized as a majority effect. Moreover, on the basis of the arguments given in Appendix I of Franke (2007) the herding effect can be called weak if $0<\phi_{x}<1$, and strong if $\phi_{x}>1$ is prevailing.

This notwithstanding, even a strong majority $x_{t-1}$ may lose its attractiveness if it is already crumbling off. This idea is captured by a positive coefficient $\phi_{\Delta x}$, which enables the negative change $\Delta_{\tau_{x}} x_{t-1}$ in this situation to have a negative impact on the feedback index $f_{t-1}$ in (6). Generally, $\phi_{\Delta x}>0$ assesses the effect that the probability of switching from pessimism to optimism is high (low) if in the recent past the number of optimistic agents has increased (decreased), from what overall level of optimism or pessimism so ever. In other words, the agents are keeping track of any changes in the current mood of the other agents and adjust their transition probabilities accordingly. Thus they view the motions of the crowd as an early warning system of future changes; they do not discard these motions as temporary but (in terms of probabilities) respond to them instantaneously. We may actually consider this effect to be herding proper: An individual sheep, to strain the metaphor and neglect the probabilistic setting, does not wait until the great majority of the flock gathers at a greener grass and then joins them, it rather follows the other sheep as soon as they begin moving. A markedly positive coefficient $\phi_{\Delta x}$ can correspondingly be taken as another manifestation of strong herding, in the sense that it captures the influence of the movements (not position) of the flock. In order to distinguish this effect from high values of the level coefficient $\phi_{x}$, i.e. from the majority effect, it may also be said that the coefficient $\phi_{\Delta x}>0$ measures a momentum effect or, more literary and still having the image of sheep in mind, a moving-flock effect. ${ }^{12}$

II The authors do not discuss the sign of their autoregressive coefficients, let alone a possible conceptual background.

I2 The expression is not meant to carry a connotation of foolishness. At least for certain breeds of sheep this behaviour is rational since it has proved to favour survival and reproduction; and at the head of a moving flock there might be a guru. Apart from that, following the herd is just one aspect of sheep-specific (or agent-specific) rationality. Readers of the German sheep crime novel "Glennkill« ("Three Bags Full« in the English translation) by Leonie Swann know that this inclination does not altogether rule out logical and individual conclusions. 
Turning to the second feedback variable in eq. (6), the basic ideas behind the effects of the output gap $y_{t-1}$ or its rate of change $\Delta_{\tau_{v}} y_{t-1}$ on the business climate are now obvious. Of course, the choice of this economic variable as a possible feedback need not be exclusive (as exemplified by Lahl and Hüfner (2003), who try several other variables in their framework). One should, however, start with a general activity variable, in level or growth rate form or both. First because many other variables of interest may be closely correlated with $y_{t-1}$ or $\Delta_{\tau_{y}} y_{t-1}$; and second because this raises the following question: Are the agents really able to predict the future course of these variables, or are their predictions rather determined by the recent past of economic activity and its changes? Or is this no contradiction at all?

In contrast to $\phi_{x}$ and $\phi_{\Delta x}$, the signs of the two coefficients $\phi_{y}$ and $\phi_{\Delta y}$ related to the output gap are a priori ambiguous. On the one hand, high levels of output or above-average growth rates may reassure optimistic agents that their current optimism is justified, and convince pessimistic agents that their fears have become obsolete. In this view, $\phi_{y}>0$, $\phi_{\Delta y}>0$. On the other hand, such a situation might also be interpreted as carrying the seeds of a future slowdown or even downturn. For example, the central bank could be expected to raise interest rates, unless it has already done so. These fears of diminishing business prospects would be reflected by negative coefficients $\phi_{y}$ or/and $\phi_{\Delta y}$. It is thus an open and interesting problem whether the two coefficients would come out significant in an empirical estimation, and if so, how they are signed.

\section{Estimation of the Climate Index}

\section{I The Empirical Data}

As mentioned in the introduction there are two leading sentiment indicators for the German economy. These surveys, which are regularly published on a monthly basis, are carried out by the ifo institute and the ZEW institute. ${ }^{13}$ While the institutes ask a series of questions and construct several indices, we focus on the respondents' expectations of the general business situation six months ahead. ${ }^{\mathrm{I}}$ This horizon is the same for both institutes, and both of them categorize the answers into the options 'better', 'worse' or 'just about the same', of which they report the difference between the percentages of the answers 'better' and 'worse'. The index is thus in relatively good concordance with our specification of the climate variable $x$ in Section 2.2.

The main difference between the two surveys are the number of participants and the economic sectors from which they are recruited. The ifo institute asks more than 7,000 business leaders and senior managers from all sectors except the financial sector (the answers are

I3 See http://www.ifo.de and http://www.zew.de. In German texts, the 'ifo institute usually presents itself in lower-case characters.

I4 Especially regarding the ifo institute, this index should not be confused with the climate or sentiment index which is occasionally referred to in the mass media, because the latter is concerned with an evaluation of the current business situation. 
weighted according to the importance of the single industries). In contrast, the ZEW survey echoes the opinion of the German financial sector, i.e., the participants are financial analysts and institutional investors from banks (comprising $77 \%$ of the sample), insurance companies, and large industrial corporations. The number of subjects contacted is about 350.

The ZEW survey has started later than the ifo survey, in December 199I. The data we are using end with 2006:6. To get a first impression of the two expectation indices, the series are plotted together in the top panel of Figure $\mathrm{I}$, where they are rescaled in order to fit into the interval between -1 and +1 . For a better comparison of the results and to be sufficiently bounded away from these end-points (cf. the derivation of eq. [2]), the factor by which the original series are multiplied is tuned such that the two indices are contained within an interval \pm 0.80 . The ifo index attains a corresponding minimum in I992:II (plotted at $t=1992.83$ ), the ZEW index a maximum in 2000:I. ${ }^{\text {Is }}$

\section{Figure I: Time Series of the Data.}
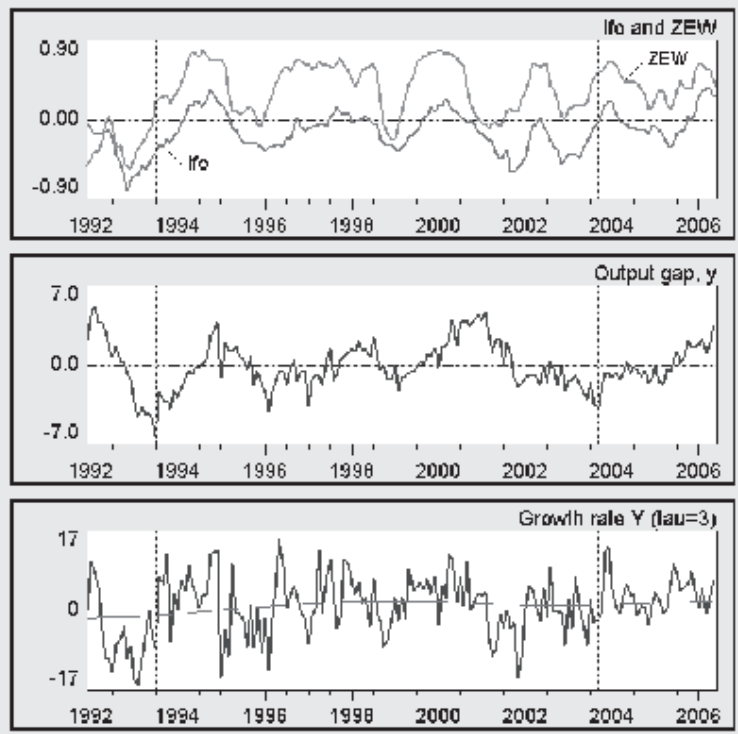

Note: The numbers in the second and third panel are percentages, where the output variable $Y$ is monthly industrial production. Detrending of log output uses the Hodrick-Prescott filter with smoothing parameter $\lambda=120,000$; the growth rates are annualized 3 -month changes. The thin line in the bottom panel is the trend growth rate implied by the filter.

At first glance the two indices exhibit a similar and clearly cyclical pattern, possibly with a tendency for the ZEW index to be slightly leading in the turning points. An obvious differ-

I5 Here and in the following it will be understood that when we refer to the 'ifo index' or 'ZEW index', the rescaled magnitudes are meant. 
ence are the levels of the two series, where from the second half of 1992 onward the ifo index runs persistently below the ZEW index; the mean values are -0.135 and 0.329 , respectively. From this descriptive point of view the ifo index can be characterized as basically pessimistic, the ZEW index as optimistic. For our structural model it may be expected that this difference finds expression in negative and positive predisposition parameters $\phi_{o}$.

The lower two panels of Figure I show the behaviour of the variable which is to represent the model's component of hetero-reference. As indicated in Section 2, we choose output for this purpose. Specifically, we work with industrial production, ${ }^{16}$ since it is the only output category available as monthly data and was also used in other work (Hüfner/Schröder 2002a,b; see, in addition, the discussion in Broyer/Savry 2002). For an export-oriented country like Germany with its weak and passive domestic demand, industrial production can, however, still be regarded as providing the basic impulses for the rest of the economy, especially for the large subsector of the business-related services (Franke/Kalmbach 2005). This output therefore contains more information about economic activity as a whole than its limited share in the national product might suggest.

The middle panel displays the output gap $y_{t}$ for this variable. It is defined as the percentage deviations of output from trend. The trend line is obtained by applying the flexible Hodrick-Prescott filter to $\log$ output, with a smoothing parameter $\lambda=120,000$. The reason for employing a roughly 8 times higher value than the conventional value of 14,400 is the variability in the implied trend growth rate (i.e., the slope of trend log output). As can be seen from the thin line in the bottom panel, trend growth still exhibits sizeable movements (though this is optically downplayed by the large fluctuations of the main series in that panel): rising from - $\mathrm{I} .6 \%$ in 1992 to $\mathrm{I} .9 \%$ in 1998 , falling to $0.9 \%$ in 2002 and increasing again to $\mathrm{I} .8 \%$ at the end of the sample. While one might wonder whether these variations still justify the notion of trend growth, the variability would be even more severe for the usual $\lambda=14,400$. Nevertheless, since the deviations of the two- or three-month output growth rates from the trend rates are fairly large and it is these deviations that will constitute the variable $\Delta_{\tau_{y}} y_{t}:=\left(y_{t}-y_{t-\tau_{y}}\right) / \tau_{y}$ from eq. ( 7$)$ in our estimations below, we can do without a stronger smoothing of the trend and maintain $\lambda=120,000$.

In order to get an impression of the changes in production, the bold line in the bottom panel of Figure I depicts the annualized three-month growth rate of output. The pattern of this series is much the same as $\Delta_{\tau_{y}} y_{t}$ with $\tau_{y}=3$. First differences of the output gap with different lags $\tau_{y}$ show a similar degree of variability, so that a priori the contribution of this variable to an econometric estimation seems quite uncertain.

A visual inspection of the comovements of the output gap with the two expectation indices shows a (near-) coincidence of the troughs of $y_{t}$ and the ifo index in 1996:2 and 1999:2, slightly lead by the ZEW index (see the vertical dashed lines in Figure I). At the two other troughs of $y_{t}$ in I993:7 and 2003:9, however, both indices are already rising for more than

I6 That is, the seasonally adjusted output of the German production sector including construction. It can be downloaded as series USNAor from http://www.bundesbank.de/statistik/statistik-zeitreihen. en.php? 
six months (at least). Conversely, at the output peak in 200I:2 the indices are on the downturn, the ZEW index being even just about to reach its next trough. Hence there is no obvious pattern of synchronized movements, contemporaneously or lagged, of the output gap and the expectation indices. ${ }^{17}$

\subsection{First Estimations of the Structural Model}

The sample period underlying our estimations of the structural model $(5)-(7)$ is the same for both the ZEW and ifo expectations. Taking account of the lags in the changes of the index and the output gap, the first month is $t=1992: 3$. The period ends with $t=2006: 6$, so that we have a total of 172 observations.

In order to get some information about which of the candidate explanatory variables may seem suitable, and with which lags, we began with a series of unconstrained linear OLS regressions of $x_{t}$ on several lagged values of itself and the output gap. Using likelihood ratio tests it turned out that the best specification is given by one lagged value of $x_{t}$ and $y_{t}$, and one first difference of these two variables. This equally holds true for the ZEW and the ifo index, though the two indices differ in the optimal lags to be chosen for $\Delta_{\tau_{x}} x_{t-1}$ and $\Delta_{\tau_{y}} y_{t-1}$. Specifically, we obtain, ${ }^{18}$

ZEW: $\tau_{x}=1, \tau_{y}=4 ;$ ifo: $\tau_{x}=3, \tau_{y}=6$

Since, so far, the formulation of the feedback index in (6) is well supported, we can turn to the structural model. Plugging (6) and (7) into (5) yields a single regression equation that can be directly estimated by nonlinear least-squares (NLS). Employing the optimal lags in (8), the basic results are collected in Table I. ${ }^{19}$

The last column RMSE of the table reports the root mean square error of the onemonth ahead predictions. These values may be related to the standard deviations $s_{x}$ of the two index series, which are,

$$
\text { ZEW: } s_{x}=0.318 ; \text { ifo: } s_{x}=0.231
$$

The corresponding $\mathrm{R}^{2}$ values are larger than 0.90. To take row 3 as an example in the first part and row $\mathrm{I}$ in the second part of the table, we have $\mathrm{R}^{2}=0.938$ for the ZEW index and $\mathrm{RMSE}=0.0793$, and $\mathrm{R}^{2}=0.925$ for the ifo index and $\mathrm{RMSE}=0.0633$. However, although these figures might suggest a good fit to the data, it should not be concealed that the forecasting abilities of the model are still limited. The greatest problem is indeed a strong pre-

I7 Statistically there are nevertheless cross-correlations $\operatorname{Corr}\left(y_{t}, x_{t-\theta}\right)=0.47$ at $\theta=6$ for the ZEW index and $\operatorname{Corr}\left(y_{t}, x_{t-\theta}\right)=0.60$ at $\theta=5$ for the ifo index, though this has not been sold as forecasting evidence so far. In fact, given the relatively smooth character of the series, the coefficients should be somewhat higher than that.

I8 Further details on the step-by-step procedure by which we arrived at (8) can be found in Franke (2007: Section 3.2)

I9 We have checked that the optimality of these lags is maintained in the nonlinear estimations. 
diction bias; that is, while the direction in which the climate index changes is mostly correctly anticipated, the amount of these changes is typically underpredicted. This problem would be a challenge to any model at a similar conceptual level.

We add that in Table $\mathrm{I}$ and the tables to follow (linear first-order) autocorrelation in the residuals is negligible. The Durbin-Watson statistic is in all cases very close to 2 , so that this issue needs no further mentioning.

The second statistic reported in Table I is two times the value $L$ of the $\log$-likelihood of the residuals $\varepsilon_{t}$ (see, e.g., Davidson/MacKinnon 2004: 403). Applying the likelihood ratio (LR) test (Davidson/MacKinnon 2004: 420f), this value will help us decide whether an estimation $\mathrm{B}$ with $r_{\mathrm{B}}$ independent variables is significantly better than a previous estimation $\mathrm{A}$ with $r_{\mathrm{A}}<r_{\mathrm{B}}$ variables. The criterion is the difference (in obvious notation) $\mathrm{LR}=2[L(\mathrm{~B})-L(\mathrm{~A})]$, which for a large enough sample is approximately distributed as chi-square with $r=r_{\mathrm{B}}-r_{\mathrm{A}}$ degrees of freedom. As can be read from any formulary, estimation B is significantly better for $r=1$ and $r=2$ at a $95 \%$ level if $\mathrm{LR}>\chi_{0.95}^{2}(1)=3.84$, or if $\mathrm{LR}>\chi_{0.95}^{2}(2)=5.99$, respectively.

Let us then apply the model to the ZEW expectations. The first row in Table I confines the model to its herding component. Besides the expected positive predisposition parameter, $\phi_{o}>0$, both herding coefficients $\phi_{x}$ and $\phi_{\Delta x}$ have the correct positive sign. However, the significantly larger likelihood statistic in the second row $(2 \mathrm{~L}=385.2)$ makes it clear that these effects should be complemented by the feedbacks from the output gap. That is, the herding mechanism has to be augmented by a mechanism of hetero-reference.

In finer detail it has here and in all other estimations to be observed that, in contrast to the growth rate coefficient $\phi_{\Delta y}$, the coefficient $\phi_{y}$ on the level of the output gap is negative. According to the interpretation at the end of Section 2, the negative sign of $\phi_{y}$ can express certain doubts of the agents that a prosperous phase of the economy will be sustained; or their hopes in a slump that the economy will be able to recover.

Even if these results make good economic sense, the standard errors in parentheses in the second row of Table I indicate that a great deficiency remains, namely, the imprecision of the estimates. If we follow the usual econometric standards then none of the six coefficients in the second row is significantly different from zero. This is especially annoying for the flexibility parameter $\nu$, since $\nu=0$ would be completely meaningless.

The high standard errors of the parameter estimates may not come as a big surprise. After all, on the right-hand side of eq. ( 5 ) we have one constant and four independent variables, which are to determine six coefficients. Without a pronounced nonlinearity there would thus be one parameter too many. In fact, a closer inspection of ( 5 ) shows that over the relevant range of the variables the curvature in the exponential function is still of little importance. ${ }^{20}$ Considering that the coefficient $\nu$ measures the overall influence of the feedback variable, whereas the $\phi$-coefficients are directly linked to the explanatory variables, we will therefore not expect that $\nu$ could be identified separately from all of the other coef-

20 See Franke (2007: Section 3.3) A side result of this investigation is that the original transition probabilities in $(4), \pi_{t}^{-/+}=\nu \exp \left( \pm f_{t}\right)$, are well specified; given the order of magnitude of the estimated 
ficients. At this stage of the analysis we have to face the problem that the structural model might be overdetermined.

Table I: NLS Estimations of the Structural Model (5)-(7).

\begin{tabular}{|c|c|c|c|c|c|c|c|c|}
\hline & $\nu$ & $\phi_{o}$ & $\phi_{x}$ & $\phi_{\Delta x}$ & $\phi_{y}$ & $\phi_{\Delta y}$ & $2 \mathrm{~L}$ & RMSE \\
\hline \multicolumn{9}{|c|}{ ZEW expectations } \\
\hline 1 & 0.110 & 0.117 & 0.78 & 2.46 & - & - & 372.0 & 0.0823 \\
\hline 2 & $\begin{array}{c}0.093 \\
(0.073)\end{array}$ & $\begin{array}{c}0.195 \\
(0.153)\end{array}$ & $\begin{array}{c}0.52 \\
(0.48)\end{array}$ & $\begin{array}{c}2.54 \\
(1.75)\end{array}$ & $\begin{array}{l}-5.42 \\
(4.49)\end{array}$ & $\begin{array}{c}32.8 \\
(25.8)\end{array}$ & 385.2 & 0.0792 \\
\hline 3 & $\begin{array}{c}0.082 \\
(0.054) \\
{[-]}\end{array}$ & 0.205 & $\begin{array}{c}0.41 \\
(0.45) \\
{[0.14]}\end{array}$ & $\begin{array}{c}2.83 \\
(1.59) \\
{[0.35]}\end{array}$ & $\begin{array}{l}-6.23 \\
(4.27) \\
{[2.05]}\end{array}$ & $\begin{array}{c}37.3 \\
(24.6) \\
{[11.0]}\end{array}$ & 384.8 & 0.0793 \\
\hline 4 & $\begin{array}{c}0.092 \\
(0.063)\end{array}$ & 0.107 & 0.71 & 2.69 & -2.78 & - & 373.8 & 0.0819 \\
\hline 5 & $\begin{array}{c}0.085 \\
(0.058)\end{array}$ & 0.174 & 0.51 & 3.11 & - & 20.8 & 375.9 & 0.0814 \\
\hline 6 & $\begin{array}{c}0.037 \\
(0.034)\end{array}$ & 0.396 & -0.17 & - & -22.07 & 81.7 & 338.2 & 0.0908 \\
\hline 7 & $\begin{array}{c}0.053 \\
(0.011)\end{array}$ & 0.342 & - & $\begin{array}{c}3.86 \\
(0.74)\end{array}$ & $\begin{array}{l}-9.05 \\
(3.05)\end{array}$ & $\begin{array}{c}57.7 \\
(14.0)\end{array}$ & 383.7 & 0.0795 \\
\hline \multicolumn{9}{|c|}{ ifo expectations } \\
\hline 1 & $\begin{array}{c}0.196 \\
(0.127)\end{array}$ & -0.044 & $\begin{array}{c}0.68 \\
(0.26)\end{array}$ & $\begin{array}{c}1.49 \\
(1.01)\end{array}$ & $\begin{array}{l}-1.68 \\
(1.36)\end{array}$ & $\begin{array}{c}20.5 \\
(13.7)\end{array}$ & 462.2 & 0.0633 \\
\hline 2 & $\begin{array}{c}0.072 \\
(0.016)\end{array}$ & -0.135 & - & $\begin{array}{c}4.02 \\
(0.89)\end{array}$ & $\begin{array}{l}-4.15 \\
(2.64)\end{array}$ & $\begin{array}{c}53.7 \\
(13.2)\end{array}$ & 460.8 & 0.0636 \\
\hline 3 & $\begin{array}{c}0.081 \\
(0.015)\end{array}$ & -0.135 & - & $\begin{array}{c}4.27 \\
(0.82)\end{array}$ & - & $\begin{array}{l}39.9 \\
(8.9)\end{array}$ & 457.1 & 0.0643 \\
\hline
\end{tabular}

Note: Numbers in parentheses are the standard errors of the estimates. Except for the first two rows in the upper part of the table, the predisposition parameter $\phi_{o}$ is determined by condition (Io), $\phi_{o}=\phi_{o}^{e}\left(\phi_{x}, \bar{x}\right)$. The lags underlying $\Delta x$ and $\Delta y$ are given in (8).

\subsection{Dealing With the Problem of Imprecise Estimates}

Before proceeding with the discussion, let us save one parameter. This is done for conceptual reasons and to simplify the computations in the estimations, though it will not yet solve the problem of overdetermination. If we take the estimates of $\phi_{o}$ and $\phi_{x}$ in the second row of Table $\mathrm{I}$ and consider the empirical mean value of the ZEW index, $\bar{x}=0.329$, then we observe that $h(\bar{x}):=(1-\bar{x}) \exp \left(\phi_{o}+\phi_{x} \bar{x}\right)-(1+\bar{x}) \exp \left(-\phi_{o}-\phi_{x} \bar{x}\right)$ is approximately zero. This rela-

$\nu$ there is no problem with the requirement that $\pi_{t}^{-/+}$must be less than unity. 
tionship motivates us to postulate directly that in the presence of $y_{t-1}=\Delta_{\tau_{y}} y_{t-1}=\Delta_{\tau_{x}} x_{t-1}=0$, the sample mean of the climate index constitutes an equilibrium of (5). The corresponding condition $h(\bar{x})=0$ can then be solved explicitly for the coefficient $\phi_{o}$. Rearranging the terms in this equation as $(1-\bar{x}) /(1+\bar{x})=\exp (-\bar{f}) / \exp (\bar{f})=\exp \left(-2 \frac{o}{f}\right)\left(\right.$ where $\left.\bar{f}=\phi_{o}+\phi_{x} \bar{x}\right)$ and taking logs, we get

$$
\phi_{o}^{e}=\phi_{o}^{e}\left(\phi_{x}, \bar{x}\right)=-\left[\frac{1}{2} \ln \frac{1-\bar{x}}{1+\bar{x}}+\phi_{x} \bar{x}\right]
$$

The superscript $e$ < indicates that this coefficient is the intercept in the feedback index that establishes $\bar{x}$ as an equilibrium point of the climate dynamics (5). Conceptually, (Io) determines the predisposition parameter of the agents from the average climate and the estimate of $\phi_{x}$. Note that the log expression is approximately $(1-\bar{x}-1)-(1+\bar{x}-1)=-2 \bar{x}$. Hence $\phi_{o}^{e} \approx\left(1-\phi_{x}\right) \bar{x}$ and, provided that $\phi_{x}$ is less than unity, a positive (negative) sample mean of the climate index is, in the structural model, indeed indicative of a predisposition of the agents toward optimism (pessimism).

For a nonlinear regression it is no additional problem to replace the cofficient $\phi_{o}$ in (5) and (6) with the value of $\phi_{o}^{e}$ in (IO) that is linked to $\phi_{x}$. The estimation result for the ZEW index is reported in the third row of Table I. Apparently, as a comparison with the likelihood in the second row shows, the requirement that the conceptual equilibrium value of $x$ coincides with the sample mean is not a very strong constraint on the data.

We can thus return to the problem of overdetermination. To begin with, the most straightforward solution to it seems to fix the parameter $\nu$ from the outside. But at what level? It would in this respect be desirable to have some evidence, possibly by way of analogy, from the psychological literature. Here we are left with an a priori plausibility of $\nu$ as the only criterion. Referring back to the transition probabilities in (4) and, for simplicity and just for the moment being, taking $\phi_{o}=0$ and $x=0$ to characterize a (hypothetical) neutral state, our most recent estimate $\nu=0.082$ has the immediate interpretation that on average an individual agent would autonomously switch every $1 / 0.082 \approx 12$ months from pessimism to optimism or vice versa. This seems an acceptable order of magnitude given the kind of expectations the agents have to form.

Exogenously fixing the flexibility parameter at $\nu=0.082$ yields, of course, the same values for the other parameters as before. However, the standard errors in square brackets in the third row of Table I make sure that the estimates become significant in this way, as it should be.

Nevertheless, before contenting ourselves with this solution and its remaining arbitrariness, let us consider the other parameters in the structural model. Setting $\phi_{y}, \phi_{\Delta y}$ and $\phi_{\Delta x}$ at some exogenous value would be even more arbitrary. On the other hand, the initial exploratory OLS regressions mentioned above suggest that putting them equal to zero would deteriorate the fit too much. The estimations in rows 4-6 in Table i fully confirm this. Apart from that, the outcome is of no great help for a more precise estimate of $\nu$, either. 
The last coefficient available is the coefficient $\phi_{x}$, which represents the majority effect in the herding mechanism. Again, we have no direct clue for a sensible non-zero level. Furthermore, $\phi_{x}$ has always been a central parameter in the theoretical models in the literature; it is, so to speak, the coefficient with which it all has begun (the first discussions of this kind of theory included only $\nu$ and $\phi_{x}$ as non-zero coefficients). Hence there is also a strong psychological barrier to let $\phi_{x}$ vanish.

The last row in the upper part of Table I surmounts this barrier - with remarkable success. First, all of the remaining coefficients are now highly significant. And second, if the likelihood is compared to that in the third row, then the deterioration in the fit is insignificant. In terms of parsimony, row 7 is therefore to be preferred to row 3 (with $\nu$ as part of the estimation). In other words, if we start from the estimation in row 7 and then consider to introduce $\phi_{x}$ as an additional coefficient, the insufficient increase of the likelihood in row 3 would advise us against this generalization of the model. In this sense the estimation in row 7 is optimal, which is emphasized by the bold face characters.

To sum up, in the estimation of the structural model (5) - (7) on the ZEW expectations index we, legitimately, decide against the majority effect and dismiss it from the model. Herding is therefore exclusively represented by what we have called the moving-flock effect $\left(\phi_{\Delta x}>0\right)$. Combining it with the hetero-reference mechanism, we settle down on the estimates presented in row 7 of Table I. Note that now the autonomous switches of an agent would be expected to occur every $1 / 0.053 \approx 19$ months, which also seems to make psychological sense. ${ }^{21}$

The estimation of the ifo expectations can proceed along the same lines. The lower part of Table I can therefore be limited to the key results. The first row is the unconstrained estimation (except for subjecting the predisposition parameter to the consistency condition (Io), $\phi_{o}=\phi_{o}^{e}\left(\phi_{x}, \bar{x}\right)$, where, as it should be for this index series, $\phi_{o}$ comes out negative). Of course, the problem of imprecise parameter estimates does not disappear, though it is remarkable that here one of the coefficients is significant. Moreover, this is just the coefficient that we have decided to discard, namely $\phi_{x}$ (see the standard error in bold face).

The second row reveals that this significance is spurious. As in the estimations before, omitting $\phi_{x}$ does not significantly lower the goodness-of-fit. A slight difference from the ZEW results is that the coefficient $\phi_{y}$ on the levels of the output gap remains insignificant.

2I In an alternative attempt, Lux (2007) goes back to the micro level, sets up the abovementioned Fokker-Planck equation in (essentially) continuous time and computes from there the conditional transitional probability densities of $x_{t}$ between two months, which can then be used for a maximum likelihood estimation. The main conceptual difference from the present approach is that in the FPE the only source of noise is the intrinsic noise, whereas we have only incorporated the extrinsic noise (apart from that, Lux works with a different scaling of the climate index). The FPE approach is potentially superior since it seeks to exploit more information, though this goes at the price of a high computational effort (8 to Io minutes for one estimation in GAUSS). Nevertheless, a reestimation of the first part of Table I with the FPE method on our data yields no significant differences (private communication with Thomas Lux and Xiaokang Wang). 
Excluding it from the model yields the estimation in the last row of the table, which we present as our upshot for the ifo expectations index.

We are thus in a position to ask for the similarities and dissimilarities in the expectation formation by the agents in the two panels of ZEW and ifo. Four observations can be made in this regard. First, the ZEW agents have a predisposition toward optimism, the ifo agents toward pessimism. Our model captures this tendency by, respectively, positive and negative estimates of the parameter $\phi_{o}$ (via the parameter $\phi_{x}$ in (IO) as well as when $\phi_{o}$ is estimated directly). While one would not need a theoretical model for such a conclusion, this does not lessen its significance.

In contrast, our second conclusion, which refers to the agents' general flexibility, would not be possible without a theoretical framework. Here we find, on the basis of our thought experiment of autonomous switches from pessimism to optimism and vice versa in a hypothetical state of equilibrium, that the ifo agents are more "flexible«. They would on average switch every $1 / 0.081 \approx 12$ months, whereas the expected frequency of the ZEW agents is I9 months (as noted above).

The third point concerns the agents' responsiveness to economic activity in the model's hetero-reference component. Although our estimation upshots show some differences in the coefficients $\phi_{y}$ and $\phi_{\Delta y}$, they should not be overrated. On the one hand, the two coefficients $\phi_{\Delta y}$ are almost the same if we compare the ZEW estimates with row 2 for the ifo expectations. On the other hand, re-estimating the ZEW expectations under the constraint $\phi_{y}=0$ leads to $\phi_{\Delta y}=34.4$, while the moving-flock coefficient increases to (only) $\phi_{\Delta x}=4.85$ (though the fit is substantially poorer). The fourth observation concerns the herding mechanism. After we have confined it to the moving-flock effect, which has been carefully justified, we see that the two groups of agents are very similar in this behavioural characteristic. Actually, the two estimates of the coefficient $\phi_{\Delta x}$ (3.86 and 4.27) can hardly be told apart.

In a very succinct way and at the risk of oversimplification we may thus summarize the estimations of our basic model as follows. Apart from a stronger predisposition towards pessimism and a somewhat higher, as we have called it, flexibility on the part of the ifo agents, the two panels of ZEW and ifo agents are not markedly different: they share the same herding mechanism and react in similar ways to the arrival of new information on economic activity.

\section{Extensions of the Basic Specification}

Regarding the feedbacks on the agents' transition probabilities one can certainly think of many additional effects that might be worth exploring. We limit ourselves to two extensions of the model. First we ask a question that becomes obvious as soon as we have the notion of two herds the agents may be following, namely, if there are also effects from one herd (or flock) to the other. More technically, we study possible cross influences of the two climate indices. The second extension of the model introduces a variable that is possibly 
taken into account by the agents but remains unobservable to the researcher or has been omitted by him or her.

\section{I Cross Effects Between the ZEW and ifo Panel}

The concept of the herd, flock or crowd has so far assumed that regarding their state of mind the agents only communicate within their own well specified group. The possibility that, besides the fundamental data, the ZEW agents may also pay attention to the sentiment of the ifo agents, and vice versa, is thus neglected.

Instead of uniting the two flocks of ZEW and ifo agents to form one homogeneous group, we continue to keep the two panels apart but now admit cross effects from one climate index on the other. A first indication that this might improve the performance of the model is given by the linear regressions conducted by Lahl and Hüfner (2003). They report a significant $t$-statistic for the ifo index if one lag of it is added to their autoregressive equation for the ZEW index. ${ }^{22}$ In the following we will investigate this extension of one index possibly impacting on the other in a more systematic way and in both directions.

The generalization of the structural model (5)-(7) is straightforward. If $x$ continues to denote the estimated index and $\xi$ is now introduced to refer to the alternative "outside« index, we only have to add $\xi_{t-1}$ and its rate of change $\Delta_{\tau_{\xi}} \xi_{t-1}$ in the specification of the feedback index $f_{t-1}$ (for a suitable lag $\tau_{\xi}$ ). The estimation equation then reads,

$$
\begin{aligned}
x_{t} & =x_{t-1}+\nu\left[\left(1-x_{t-1}\right) \exp \left(f_{t}\right)-\left(1+x_{t-1}\right) \exp \left(-f_{t}\right)\right]+\varepsilon_{x, t} \\
f_{t-1} & =\phi_{0}+\phi_{x} x_{t-1}+\phi_{\Delta x} \Delta_{\tau_{x}} x_{t-1}+\phi_{y} y_{t-1}+\phi_{\Delta y} \Delta_{\tau_{y}} y_{t-1}+\phi_{\xi} \xi_{t-1}+\phi_{\Delta \xi} \Delta_{\tau_{\xi}} \xi_{t-1} \\
\phi_{o} & =\phi_{o}^{e}\left(\phi_{x}, \bar{x}\right) \text { from (IO) }
\end{aligned}
$$

Similar to the preparation of the results in Table I for the basic model, we have indeed checked that, if at all, only two lags of the alternative index are significant, the first one being $t-1$. Equation ( 13 ) collects the optimal lags in the rates of change of $\xi$ (which yield the highest likelihood), together with those established for the other variables:

$$
\begin{array}{llll}
\tau_{x}=1 & \tau_{y}=4 & \tau_{\xi}=2 & \text { for } x=\mathrm{ZEW} \text { index } \\
\tau_{x}=3 & \tau_{y}=6 & \tau_{\xi}=1 & \text { for } x=\text { ifo index }
\end{array}
$$

Table 2 presents the main estimations of equations (II)-(I3). To begin with the ZEW index, the first row in the upper part of the table reproduces row 3 from Table I. Its likelihood serves as a benchmark to assess whether the ifo agents have a significant influence on the expectations formed by the ZEW agents, through what direct or indirect channels so ever.

22 Incidentally, including instead the first differences of the (nonstationary) U.S. consumer confidence index showed a similar effect. 
Table 2: Estimations of Cross Effects, Equations (II)-(I3).

\begin{tabular}{|c|c|c|c|c|c|c|c|c|c|}
\hline & $\nu$ & $\phi_{x}$ & $\phi_{\Delta x}$ & $\phi_{y}$ & $\phi_{\Delta y}$ & $\phi_{\xi}$ & $\phi_{\Delta \xi}$ & $2 \mathrm{~L}$ & RMSE \\
\hline \multicolumn{10}{|c|}{ ZEW expectations } \\
\hline 1 & $\begin{array}{c}0.082 \\
(0.054)\end{array}$ & $\begin{array}{c}0.41 \\
(0.45)\end{array}$ & $\begin{array}{c}2.83 \\
(1.59)\end{array}$ & $\begin{array}{l}-6.23 \\
(4.27)\end{array}$ & $\begin{array}{c}37.3 \\
(24.6)\end{array}$ & - & - & 384.8 & 0.0793 \\
\hline 2 & $\begin{array}{c}0.105 \\
(0.070)\end{array}$ & $\begin{array}{c}0.71 \\
(0.34)\end{array}$ & $\begin{array}{c}1.66 \\
(1.08)\end{array}$ & - & - & $\begin{array}{c}0.19 \\
(0.17)\end{array}$ & $\begin{array}{c}2.67 \\
(1.77)\end{array}$ & 386.2 & 0.0790 \\
\hline 3 & $\begin{array}{c}0.088 \\
(0.062)\end{array}$ & $\begin{array}{c}0.42 \\
(0.54)\end{array}$ & $\begin{array}{c}1.94 \\
(1.25)\end{array}$ & $\begin{array}{l}-3.49 \\
(3.45)\end{array}$ & $\begin{array}{c}29.5 \\
(21.5)\end{array}$ & $\begin{array}{l}-0.15 \\
(0.21)\end{array}$ & $\begin{array}{c}2.64 \\
(1.84)\end{array}$ & 394.7 & 0.0770 \\
\hline 4 & $\begin{array}{c}0.053 \\
(0.011)\end{array}$ & - & $\begin{array}{c}3.86 \\
(0.74)\end{array}$ & $\begin{array}{l}-9.05 \\
(3.05)\end{array}$ & $\begin{array}{c}57.7 \\
(14.0)\end{array}$ & - & - & 383.7 & 0.0795 \\
\hline 5 & $\begin{array}{c}0.062 \\
(0.011)\end{array}$ & - & $\begin{array}{c}2.59 \\
(0.66)\end{array}$ & $\begin{array}{l}-5.65 \\
(2.71)\end{array}$ & $\begin{array}{c}42.5 \\
(12.3)\end{array}$ & - & $\begin{array}{c}3.47 \\
(1.09)\end{array}$ & 393.9 & 0.0772 \\
\hline \multicolumn{10}{|c|}{ ifo expectations } \\
\hline 1 & $\begin{array}{c}0.196 \\
(0.127)\end{array}$ & $\begin{array}{c}0.68 \\
(0.26)\end{array}$ & $\begin{array}{c}1.49 \\
(1.01)\end{array}$ & $\begin{array}{l}-1.68 \\
(1.36)\end{array}$ & $\begin{array}{c}20.5 \\
(13.8)\end{array}$ & - & - & 462.2 & 0.0633 \\
\hline 2 & $\begin{array}{c}0.122 \\
(0.142)\end{array}$ & $\begin{array}{c}0.64 \\
(0.54)\end{array}$ & $\begin{array}{c}2.22 \\
(2.58)\end{array}$ & - & - & $\begin{array}{c}0.05 \\
(0.10)\end{array}$ & $\begin{array}{c}0.37 \\
(0.50)\end{array}$ & 448.6 & 0.0659 \\
\hline 3 & $\begin{array}{c}0.200 \\
(0.136)\end{array}$ & $\begin{array}{c}0.73 \\
(0.27)\end{array}$ & $\begin{array}{c}1.20 \\
(0.86)\end{array}$ & $\begin{array}{l}-1.70 \\
(1.35)\end{array}$ & $\begin{array}{c}20.0 \\
(13.8)\end{array}$ & $\begin{array}{l}-0.00 \\
(0.04)\end{array}$ & $\begin{array}{c}0.22 \\
(0.21)\end{array}$ & 464.1 & 0.0630 \\
\hline 4 & $\begin{array}{c}0.081 \\
(0.015)\end{array}$ & - & $\begin{array}{c}4.27 \\
(0.82)\end{array}$ & - & $\begin{array}{l}39.9 \\
(8.9)\end{array}$ & - & - & 457.1 & 0.0643 \\
\hline 5 & $\begin{array}{c}0.075 \\
(0.016)\end{array}$ & - & $\begin{array}{c}3.94 \\
(0.88)\end{array}$ & - & $\begin{array}{l}42.2 \\
(9.7)\end{array}$ & - & $\begin{array}{c}0.54 \\
(0.45)\end{array}$ & 458.8 & 0.0639 \\
\hline
\end{tabular}

The second row of the table replaces the feedback from the output gap with the feedback from the ifo index. With respect to the goodness-of-fit they bring about, the two effects are about equally strong. Combining the two effects in the third row shows that each of the two effects accomplishes a significant improvement over the isolated contribution of the other.

This characterization does not yet take the precision of the single coefficients into account. Precision is again obtained by discarding the majority effect in the herding mechanism, setting $\phi_{x}=0$. For a better comparison with the basic model, the fourth row in the table repeats the estimation on which we have settled down in row 7 of Table I, where all of the coefficients have come out highly significant. This feature is fully maintained in row 5 of Table 2 if the changes $\Delta_{\tau \xi} \xi_{t-1}$ of the ifo climate are included as the only additional feedback variable. The coefficient $\phi_{\Delta \xi}$ on the latter proves to be highly significant, too, while, as already indicated by the high standard error in row 3 , the level effect from $\xi_{t-1}$ would be rather insubstantial. Hence, again, the changes in the agents' attitudes are more important than their current level. Since also the likelihood increases considerably from row 4 to row 5 , we can conclude that the augmented model (II)-(I3) is strongly supported by the data 
and, with the variables selected, provides a powerful description of the expectation formation process of the ZEW agents.

The lower part of Table 2 documents the influence of the ZEW index on the ifo index, or rather the non-existence of such an influence. The first row reproduces the first row of the ifo estimations in Table I. In row 2 and 3 it is seen that neither replacing the output gap nor combining it with the ifo index is of any relevance. The same conclusion has to be drawn if the estimations are restricted to the most significant coefficients. Hence also in the extended framework it turns out that for the ifo index the estimation in row 3 of Table I, which is repeated in row 4 of Table 2 , remains the result that we can offer to take home. Of course, it cannot be ruled out that there might be other variables that have a greater impact on the ifo expectations. Considering, however, the relatively low RMSE and the corresponding $\mathrm{R}^{2}$, which amounts to 0.922 , the explanatory power of this estimation is already remarkable.

\subsection{Estimation With an Unobservable Variable}

In this section we try a more elaborate estimation approach than the previous nonlinear least-squares minimization. In principle, the approach can account for the effects that so far have been omitted. It does this by lumping them all together in one single dynamic variable $a_{t}$ that is added to our feedback index $f_{t-1}$, where $a_{t}$ is stochastic as well as unobservable. The promise of "in principle« is qualified by the need to specify a stochastic law of motion for that variable, whose parameters will be part of the estimation. To guard against the possible criticism of arbitrariness, which to deal with would require a careful investigation of a battery of alternative cases, we will here content ourselves with a most parsimonious form.

Let us assume that the unobservable variable $a_{t}$ follows a first-order autoregressive process with an autocorrelation coefficient $\rho$. Let $\sigma_{a}$ and $\sigma_{x}$ be the standard deviations of the two random terms that impact on $a_{t}$ and the climate index $x_{t}$, respectively, and reserve the notation $\eta_{a}, \eta_{x}$ to (independent) draws from the standard normal distribution (with mean zero and variance one). The system to be estimated then reads,

$$
\begin{aligned}
x_{t} & =x_{t-1}+\nu\left[\left(1-x_{t-1}\right) \exp \left(f_{t-1}+a_{t}\right)-\left(1+x_{t-1}\right) \exp \left(-f_{t-1}-a_{t}\right)\right]+\sigma_{x} \eta_{x, t} \\
a_{t} & =\rho a_{t-1}+\sigma_{a} \eta_{a, t} \\
f_{t-1} & =\phi_{0}+\phi_{x} x_{t-1}+\phi_{\Delta x} \Delta_{\tau_{x}} x_{t-1}+\phi_{y} y_{t-1}+\phi_{\Delta y} \Delta_{\tau_{y}} y_{t-1}+\phi_{\xi} \xi_{t-1}+\phi_{\Delta \xi} \Delta_{\tau_{\xi}} \xi_{t-1} \\
\phi_{o} & =\phi_{o}^{e}\left(\phi_{x}, \bar{x}\right) \text { from (IO), the lags } \tau_{x}, \tau_{y}, \tau_{\xi} \text { from (I3) }
\end{aligned}
$$

The variable $a_{t}$ can be conceived of as representing a general composite variable made up of additional fundamental data such as wages, interest rates, exchange rates, political news, etc., which so far have been neglected. Alternatively, or additionally, $a_{t}$ may comprise the measurement errors that we have discussed when introducing the basic equations (5)-(7). $a_{t}$ is thus a very convenient "catch-all « variable which, however, is presupposed to evolve in a fairly regular manner. 
An estimation of model (I4)-(I6) uses the Kalman filter to set up a likelihood function, which is to be maximized. Under the assumption of normal distributions, the Kalman filter is concerned with the prior and posterior probability densities of the unobservable state in each period $t$, that is, with their means and variances. In essence, the Kalman filter is an optimal updating algorithm for them, given the parameters in the equations (which include the standard deviations $\sigma_{x}$ and $\sigma_{a}$ ). Since the procedure is based on linear relationships but (I4) is nonlinear, one has to work with linear approximations. They constitute the extended Kalman filter, as it is more precisely called. On this basis a likelihood function for the observable variable $x_{t}$ can be set up, which is then to be maximized across the parameters of interest. ${ }^{23}$

A degenerate case of this likelihood maximization is obtained by fixing $a_{t}=0, \sigma_{a}=0$ in (I5). It is important to note that it is equivalent to the nonlinear least-squares estimation from above. This feature, in particular, implies that in order to evaluate the goodness-of-fit of the different methods, the likelihood values reported in the previous tables can be directly compared to the values resulting from the estimation of (I4)-(I6).

The question of whether an estimation of (I4)-(I6) can outperform the previous results has two clear answers: 'no ' for the ifo index, and 'yes f for the ZEW index. We abstain from a documentation of all the failures when applying (I4)-(I6) to the ifo index and concentrate on the main results for the ZEW index. They are reported in Table 3, where in two blocks the estimations are carried out without and with incorporating the cross effects from the alternative index, i.e., from the ifo index. To begin with the simpler specification, the first row reproduces our upshot of the estimation of the basic model, row 7 from Table I. Freezing $a_{t}=0$ in (I5), we get a standard deviation $\sigma_{x}=0.0795$ in eq. (I4) and, as just asserted, the same likelihood as in the NLS estimation.

The second row in Table 3 shows that the introduction of the unobserved variable $a_{t}$ leads to a marked improvement. The serial correlation in $a_{t}$ is quite low and cancelling it, $\rho=0$, does not cause a significant deterioration in the likelihood. The bold face figures in the third row of the table are thus the optimal result that we get for the ZEW climate, when the feedbacks in the transition probabilities are limited to the index itself and the output gap.

Comparing row 3 to row $\mathrm{I}$, it is seen that the coefficient estimates do not differ very much. The standard errors for $\phi_{\Delta x}, \phi_{y}, \phi_{\Delta y}$ are slightly better in the new version, while the precision of the flexibility coefficient $\nu$ has almost doubled. These positive features have become possible by a changing role of the random perturbations. With $\sigma_{x} \approx 0$ they no longer show up as an additive term to the climate's aggregate adjustment equation, where they summarize influences from outside the model and perhaps also correct for possible misspecifications in the functional form. Instead, the perturbations are directly connected to the feedback index, which means they can be regarded as occurring within the modelling framework. It is furthermore remarkable that they are serially uncorrelated. Hence if there are important variables omitted by us, then at least they do not behave too regularly. These

23 A self-contained description of this method suited to the present case is given in Franke (2007: appendix A.3) 
observations underline the explanatory power of the model's transition probabilities as they are set out, and our choice of the variables specifying the feedback index.

Table 3: Kalman Filter Estimations of (I4)-(I6) for the ZEW Index.

\begin{tabular}{ccccccccccc}
\hline & $\nu$ & $\phi_{x}$ & $\phi_{\Delta x}$ & $\phi_{y}$ & $\phi_{\Delta y}$ & $\phi_{\Delta \xi}$ & $\rho$ & $\sigma_{a}$ & $\sigma_{x}$ & $2 \mathrm{~L}$ \\
\hline 1 & 0.053 & - & 3.86 & -9.05 & 57.7 & - & - & - & 0.0793 & 383.7 \\
& $(0.011)$ & & $(0.74)$ & $(3.05)$ & $(14.0)$ & & & & & \\
2 & 0.058 & - & 2.84 & -10.47 & 61.5 & -- & 0.15 & 0.672 & 0.0001 & 394.3 \\
& $(0.007)$ & & $(1.11)$ & $(3.09)$ & $(13.9)$ & & $(0.08)$ & $(0.140)$ & & \\
3 & 0.055 & - & 3.84 & -9.91 & 64.5 & - & - & 0.705 & 0.0000 & 393.1 \\
& $(0.006)$ & & $(0.59)$ & $(2.81)$ & $(13.2)$ & & & $(0.076)$ & & \\
4 & 0.072 & 0.34 & 3.16 & -7.90 & 47.6 & - & - & 0.560 & 0.0000 & 394.4 \\
& $(0.010)$ & $(0.18)$ & $(0.55)$ & $(2.46)$ & $(13.5)$ & & & $(0.070)$ & & \\
\multirow{2}{*}{5} & 0.062 & - & 2.59 & -5.65 & 42.5 & 3.47 & - & - & 0.0772 & 393.9 \\
& $(0.011)$ & & $(0.66)$ & $(2.71)$ & $(12.3)$ & $(1.09)$ & & & & \\
\multirow{2}{*}{6} & $\mathbf{0 . 0 5 9}$ & - & 2.62 & -6.68 & 50.8 & 4.40 & - & 0.625 & 0.0000 & 409.5 \\
& $(0.006)$ & & $(0.57)$ & $(2.64)$ & $(12.5)$ & $(0.07)$ & & $(0.068)$ & & \\
7 & 0.061 & 0.06 & 2.56 & -6.46 & 48.3 & 4.20 & - & 0.605 & 0.0000 & 409.5 \\
& $(0.008)$ & $(0.20)$ & $(0.57)$ & $(2.61)$ & $(14.3)$ & $(0.07)$ & & $(0.071)$ & & \\
\hline
\end{tabular}

Technically, the changing place where the random forces take effect indicates the benefits from the nonlinear structure of the model. It appears that the superior results from the estimation of (I4)-(I6) can be ascribed to the curvature, however slight, in the exponential function, and the variability of the unobserved perturbations $a_{t}$ (brought about by $\sigma_{a} \eta_{a, t}$ ) is able to exploit this feature.

Things are very similar when the variable $\Delta_{\tau_{\xi}} \xi_{t-1}$ enters the feedback index in addition. The fifth row in Table 3 reiterates the final result from Table 2, and row 6 in Table 3 points out the improvement achieved by (I4)-(I6), which, in terms of the likelihood, is even stronger than before. Particularly astonishing is the minimal standard error of the estimate of $\phi_{\Delta \xi}$. Again, the function of the random perturbations of $x_{t}$ is completely taken over by the random forces impacting directly on the transition probabilities, and again they do not exhibit significant autocorrelation.

Finally, we come back to the majority effect in the herding mechanism and the corresponding coefficient $\phi_{x}$. Could it be that in the new approach, which can better exploit the model's nonlinearities, this effect has a greater role to play? The estimations in row 4 and 7 of Table 3 disprove this idea, as in both cases the increase in the likelihood is far from significant (despite the relatively low standard error of $\phi_{x}$ in row 4 ). The validity of the generalized model (I4)-(I6) is furthermore accentuated by the fact that now, although with the reintroduction of $\phi_{x}$ we could have "one parameter too many « as discussed in Section 3, the estimates of the other coefficients maintain their precision. 
To sum up, the emphasized rows 3 and 6 in Table 3 are indeed an adequate description of how the agents in the ZEW panel form their optimistic and pessimistic expectations about the economy for the next six months, qualitatively (regarding especially the choice of the selected variables) as well as quantitatively (regarding the reaction intensities). In the estimations of the model for the ifo agents, the exogenous random forces have turned out to play a different role, since here they are disconnected from the feedback index and act on the aggregate index $x_{t}$ directly. The good estimation results (row 3 in Table I and row 4 in Table 2) show that nevertheless this panel, too, provides sound support for our modelling approach. ${ }^{24}$

\section{Conclusion}

The paper has designed a population of agents taking one of two opposite attitudes as, for example, optimism and pessimism. Their switches from one attitude to the other are most suitably modelled by transition probabilities. In this way a macroscopic adjustment equation for the aggregate climate in the population can be derived, whose stochastic diffusion elements become negligible if the population is large. Various feedbacks can be incorporated into this dynamic equation by specifying the individual transition probabilities as functions of (a) the current level of the climate index, (b) its rate of change, and (c) other fundamental data. Feedbacks (a) and (b) can be said to constitute a herding dynamics, while (c) allows the agents to take external norms into account that may have an additional influence on their attitudes.

We would like to present our approach as an alternative to the mainstream macroeconomics founded on the representative agent. It starts out from many heterogeneous agents who constantly change their state of mind in both directions. Their aggregate opinion, or climate, can nevertheless be conveniently studied at the macroeconomic level, just as in any other heterodox and feedback-guided modelling framework. Since an explicit microeconomic basis has been provided, we do not need to invoke a "typical« agent to whom also most heterodox economists (explicitly or implicitly) refer in their discussion of behavioural patterns.

Postulating feedbacks from appropriate macroeconomic fundamentals such as aggregate output, wages or interest rates, the climate adjustment equation can be easily combined with other building blocks from the toolbox of feedback-guided macro modelling. This has been shown elsewhere in a parsimonious theoretical model of the economy (Franke 2008). It develops two- and three-dimensional Goodwinian income distribution dynamics in which one can legitimately speak of "animal spirits « determining investment demand of firms and thus economic activity. By contrast, the present paper is concerned with the em-

24 One obvious reason why the approach (I4)-(I6) does not work for the ifo agents could be that the specification of the stochastic law for the unobservable variable is too simple, and that a broader ARMA specification might be more successful. 
pirical validity of the modelling design. While in the aforementioned macro dynamics the climate index is an unobserved variable, one can alternatively use survey expectations by people from the business and financial world about the general business situation over the next six months as a proxy for it. The law that governs the changes in the climate can then be estimated directly. In Germany there are monthly data on two such surveys by the ifo and ZEW institutes that are well suited for this task, especially since their indices are constructed in a similar way to our two-state attitudes of the agents.

Choosing the output gap and its rate of change as the agents' fundamental news and applying the thus specified model to this kind of data proved rather successful. The estimated coefficients made economic (and psychological) sense and were significant or even highly significant. Most importantly, we could identify a significant herding mechanism, which is of roughly equal intensity in the two panels. It is in this respect remarkable that the individual agents tend to change their attitude not so much in response to the current level of the majority beliefs, but in response to their most recent changes. That is, we can state that in both panels the formation of expectations is characterized by strong herding, in that figuratively speaking the agents do not just join the crowd but follow each single motion of the crowd. An additional finding is that the ZEW agents from the financial sector are also influenced by the motions of the "crowd « of the ifo agents, who are leaders and senior managers from the business sector, whereas there are no significant cross effects in the other direction. All these results demonstrate a substantial explanatory power of the theoretical model.

It would, of course, be desirable to test the approach with other similarly constructed survey data on the one hand, and on the other hand to estimate an entire macroeconomic system with our aggregate adjustment equation as a constituent part, where the climate index itself remains unobserved. It is furthermore straightforward to extend the model to three states for the agents' attitude: optimistic, pessimistic and indifferent, say. If in addition to the difference between optimistic and pessimistic agents also data on the share of indifferent agents are available, one could try an even harder test of the microfounded modelling approach put forward here.

\section{References}

Alfarano, S., Lux, T. (2007): A noise trader model as a generator of apparent power laws and long memory, Macroeconomic Dynamics, II (Supplement), 80-IoI.

Alfarano, S., Lux, T., Wagner, F. (2005): Estimation of agent-based models: The case of an asymmetric herding model, Computational Economics, 26, 19-49.

Broyer, S., Savry, G. (2002): German leading indicators: Which one should be monitored, in: CDC IXIS Capital Markets Flash, No. 2002-38, URL: http://www.zew.de/de/ publikationen/Konjunkturerwartungen/flazoo2-38gb.pdf.

Davidson, R., MacKinnon, J.G. (2004): Econometric theory and methods, New York: Oxford University Press. 
Flaschel, P., Franke, R., Semmler, W. (1997): Dynamic macroeconomics: Instability, fluctuations, and growth in monetary economies, Cambridge (Mass.): MIT Press.

Franke, R. (2007): Estimation of a microfounded herding model on German survey expectations: Extended version, University of Kiel, Working Paper, URL: www.bwl.uni-kiel. de/vwlinstitute/gwif/downloads.php?lang=en.

Franke, R. (2008): Microfounded animal spirits and Goodwinian income distribution dynamics, in: Flaschel, P., Landesmann, M. (eds.), Mathematical economics and the dynamics of capitalism. Goodwin's legacy continued, London: Routledge, 239-254.

Franke, R., Asada, T. (1994): Expectations dynamics in a Keynes-Goodwin model of the business cycle, Journal of Economic Behavior and Organization, 24, 273-295.

Franke R., Kalmbach, P. (2005): Structural change in the manufacturing sector and its impact on business-related services: An input-output study for Germany, Structural Change and Economic Dynamics, I6, 467-488.

Haag, G., Weidlich, W., Mensch, G. (1987): The Schumpeter clock, in: Batten, D. et al. (eds.), Economic evolution and structural adjustment, Berlin: Springer.

Hommes, C.H. (2006): Heterogeneous agent models in economics and finance, in: Tesfatsion, L., Judd, K.L. (eds.), Handbook of computational economics, Vol. 2: Agent-based computational economics, Amsterdam: Elsevier.

Hüfner, F.P., Schröder, M. (2002a): Forecasting economic activity in Germany: How useful are sentiment indicators?, ZEW Discussion Paper, No. 02-56.

Hüfner, F.P., Schröder, M. (2002b): Prognosegehalt von ifo-Geschäftserwartungen und ZEW Konjunkturerwartungen: Ein ökonometrischer Vergleich, Jahrbücher für Nationalökonomie und Statistik, 223, 316-336.

Kirman, A. (1993): Ants, rationality, and recruitment, Quarterly Journal of Economics, Io8, I37-I56.

Kraft, P., Landes, T., Weise, P. (1986): Dynamic aspects of a stochastic business cycle model, Methods of Operations Research, 53, 445-453.

Lahl, D., Hüfner, F. (2003): What determines the ZEW indicator?, ZEW Discussion Paper, No. $03-48$.

Lux, T. (1995): Herd behaviour, bubbles and crashes, Economic Journal, I05, 88I-896.

Lux, T. (1997): Time variation of second moments from a noise trader/infection model, Journal of Economic Dynamics and Control, 22, I-38.

Lux, T. (1998): The socio-economic dynamics of speculative markets: Interacting agents, chaos, and the fat tails of return distributions, Journal of Economic Behavior and Organization, 33, I43-165.

Lux, T. (2007): Parameter estimation for stochastic models of interacting agents: An approximate ML approach via numerical solutions of transitional densities, University of Kiel, Working Paper.

Orléan, A. (1989): Mimetic contagion and speculative bubbles, Theory and Decision, 27, 6392.

Orléan, A. (1995): Bayesian interactions and collective dynamics of opinion: Herd behavior and mimetic contagion, Journal of Economic Behavior and Organization, 28, 257-274. 
Stadler, V. (200I): Recovery in Germany? What sentiment indicators are telling us, Hypo Vereinsbank PolicyWatch Economics, No. 07/o9/oI, URL: http://www.zew.de/de/ publikationen/Konjunkturerwartungen/hvb-paper.pdf.

Taylor, L., O’Connell, S. (I985): A Minsky crisis, Quarterly Journal of Economics, Ioo, 87I886.

Weidlich, W., Braun, M. (1992): The master equation approach to nonlinear economics, Journal of Evolutionary Economics, 2, 233-265.

Weidlich, W., Haag, G. (1983): Concepts and models of a quantitative sociology. The dynamics of interacting opulations, Berlin: Springer.

Weise, P., Kraft, M. (1988): Cumulative processes, disproportionalities, and Spiethoff's theory of the business cycle, in: Flaschel, P., Krüger, M. (eds.), Recent approaches to economic dynamics, Frankfurt a.M.: P. Lang, 3-16. 